\title{
Application of the Convergent Beam Imaging (CBIM) Technique to the Analysis of Crystal Defects
}

\author{
Jean-Paul Morniroli $\left({ }^{1}\right)$, Patrick Cordier $\left({ }^{1}\right)$, Éric Van Cappellen $\left({ }^{2}\right)$, \\ Jin Min Zuo $\left({ }^{3}\right)$ and John Spence $\left({ }^{3}\right)$ \\ $\left({ }^{1}\right)$ LSPES, URA CNRS 234, Université de Lille I, 59655 Villeneuve d'Ascq Cedex, France \\ $\left({ }^{2}\right)$ Philips Electron Optics B.V. Bldg AAE, Achtseweg Noord, PO Box 218, 5600 MD Eindhoven, \\ Pays-Bas \\ $\left({ }^{3}\right)$ Department of Physics, Arizona State University, Tempe, AZ 85287, USA
}

(Received September 25; accepted October 10, 1997)
PACS.61.14.Rq - Other electron diffraction and scattering techniques for structure analysis
PACS.61.14.Lj - Convergent-beam electron diffraction, selected-area electron diffraction, nanodiffraction

PACS.61.72.Dd - Experimental determination of defects by diffraction and scattering

\begin{abstract}
It is shown how the technique of Convergent Beam IMaging (CBIM), proposed by Humpreys et al. in 1988, can be useful for the analysis of crystal defects such as dislocations in Garnet. It is also shown how the original technique can be greatly improved by using an objective aperture, a very small spot size and an energy filter. With these experimental conditions, the quality of the CBIM patterns is nearly as good as the quality of LACBED patterns, with which they are compared.
\end{abstract}

\section{Introduction}

The LACBED technique is now a well-known technique. It was proposed by Tanaka in 1980 [1] to prevent the superimposition of the diffracted and transmitted discs in CBED patterns which occurs when the convergence semi-angle $\alpha$ of the incident electron beam becomes larger than the Bragg angles $\theta_{\mathrm{B}}$. In addition to the rocking curve information of CBED, the LACBED has proved to have new information arising from the fact that it is a defocus method, in which the electron probe is not focused directly on the sample.

As a result, the LACBED patterns contain information on both reciprocal and real spaces, which allows the mapping of the diffraction pattern simultaneously with the corresponding image. Cherns and Preston have shown that this mapping can be very useful for the analysis of dislocations since it gives typical effects directly connected with the Burgers vector of the dislocations [2]. Burgers vectors were first determined from CBED patterns by Carpenter and Spence [3], who described methods for computing these patterns in two and n-beam theory.

The technique has been extensively used for the analysis of crystal defects: perfect and partial dislocations $[4,5]$, dislocations in grain boundaries [6], dislocations in quasicrystals [7], 
stacking faults [8]. A review of applications, including the use of sub-nanometer field emission probes and coherence effects, can be found in Spence and Zuo [9], together with practical instructions for setting up the LACBED method. In addition, the LACBED patterns have a very good quality thanks to the selected area aperture which acts as an energy filter and removes most of the inelastically scattered electrons [10].

Nevertheless, the technique has some disadvantages. Since it is a defocus method, it requires crystal sizes larger than $0.2-0.5 \mu \mathrm{m}$. For the analysis of defects there are two other main disadvantages:

- the specimen is not located at the eucentric height in the electron microscope;

- the image of the defect analysed is out of focus, since it is a "shadow" image, not observed in the image plane but in the back focal.plane of the objective lens.

This means that great experimental difficulties can be encountered during tilt experiments. This is especially the case when the dislocation contrast is poor or when dislocations are too close together. It can be very tedious to localize the dislocations of interest with confidence. Indeed, Xin and Duan [11] have proposed the use of dark field LACBED to visualize the dislocations, but they also show that this technique is restricted to some special cases.

The CBIM (Convergent Beam IMaging) technique, proposed by Humphreys et al. in 1988 $[12,13]$, is an alternative to LACBED. In this technique, the specimen is situated at the eucentric height and the image is in focus (object conjugate to detector). On the other hand, the superimposed diffraction pattern is out of focus, but it can remain of sufficiently good quality provided very small spot sizes are used. Until now, this technique was mainly used for the analysis of strains at grain boundaries.

In this paper, the CBIM technique is discussed in detail and compared with the LACBED technique. The role of various experimental parameters such as the $\mathrm{C}_{2}$ focus setting, the spot size, the objective aperture and the energy filtering are studied. The application of CBIM to the characterization of crystal defects is suggested, and illustrated in the case of dislocations in garnet.

\section{Description of the LACBED Technique}

In LACBED (Fig. 1a), an incident electron beam with a semi-angle of convergence $\alpha$ in the range of 1 to $5^{\circ}$ and a spot size $S$ in the range of 5 to $50 \mathrm{~nm}$ is focused on the object plane of the objective lens.

The incident beam can be considered as being composed of elementary incident beams having all the orientations within the illumination cone.

For the sake of simplicity, let us consider a single crystal sample with a perfect plane parallel geometry. The specimen is not located at its normal position in the object plane of the objective lens (which usually corresponds to the eucentric height), but it is raised (or lowered) from this plane by a distance $\Delta h$.

Among all the elementary incident beams, the ones directed along AE are exactly at the Bragg position for the $(h k l)$ lattice planes. They interact with the specimen, at $\mathrm{A}^{\prime}$, to give $h k l$ diffracted and transmitted beams producing, an excess (bright) $h k l$ line and a deficiency (dark) $h k l$ line in the back focal plane of the objective lens.

Since the beam convergence $2 \alpha$ is very large as compared with the Bragg angle $\theta_{\mathrm{B}}$, electron diffraction can also occur on the other side of the $(h k l)$ lattice planes, at $\mathrm{B}^{\prime}$, for the elementary incident beams directed along BE. In the back focal plane, they produce, two $\bar{h} \bar{k} \bar{l}$ excess and deficiency lines. 


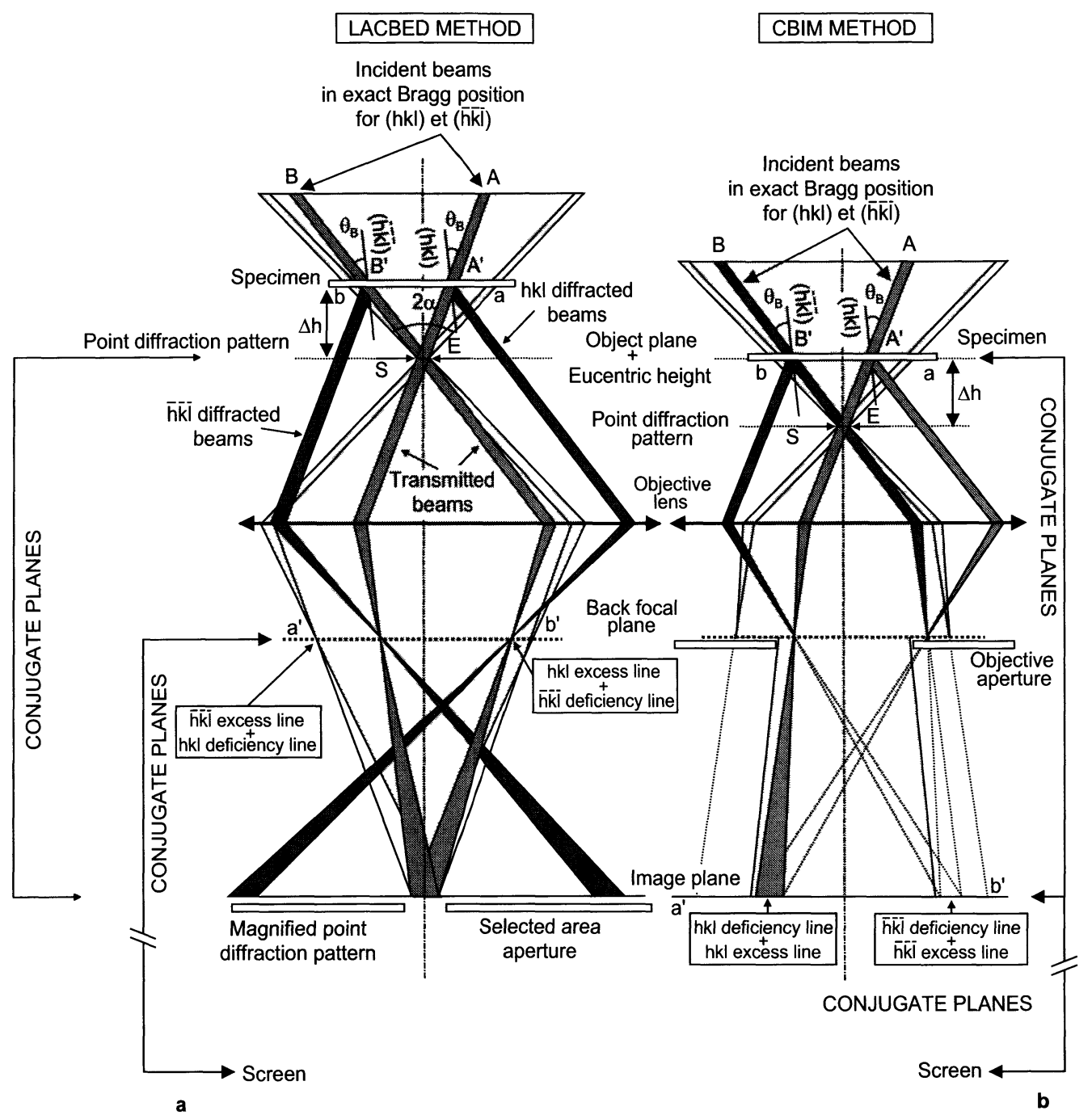

Fig. 1. - Comparison of the LACBED and CBIM techniques. a) LACBED technique. The two $h k l$ deficiency and $\bar{h} \bar{k} \bar{l}$ excess lines as well as the two $\bar{h} \bar{k} \bar{l}$ deficiency and $h k l$ excess lines are superimposed in the back focal plane of the objective lens. This plane is conjugated with the screen and the selected area aperture can remove these line superimpositions. b) CBIM technique. The two $h k l$ deficiency and excess lines as well as the two $\bar{h} \bar{k} \bar{l}$ deficiency and excess lines are superimposed in the image plane of the objective lens. This plane is conjugated with the screen and the objective aperture can remove these line superimpositions.

In this plane, the $h k l$ excess line is superimposed with the $\bar{h} \bar{k} \bar{l}$ deficiency line and the $\bar{h} \bar{k} \bar{l}$ excess line is superimposed with the $h k l$ deficiency line. Since the excess lines are bright and the deficiency ones are dark, the overall contrast is very poor.

A solution to this problem was suggested by Tanaka [1].

By raising the specimen over a distance $\Delta h$, the transmitted beams, the $h k l$ diffracted beams and the $\bar{h} \bar{k} \bar{l}$ diffracted beams are separated in the object plane of the objective lens 


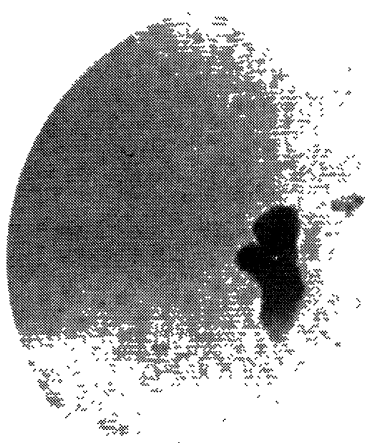

a
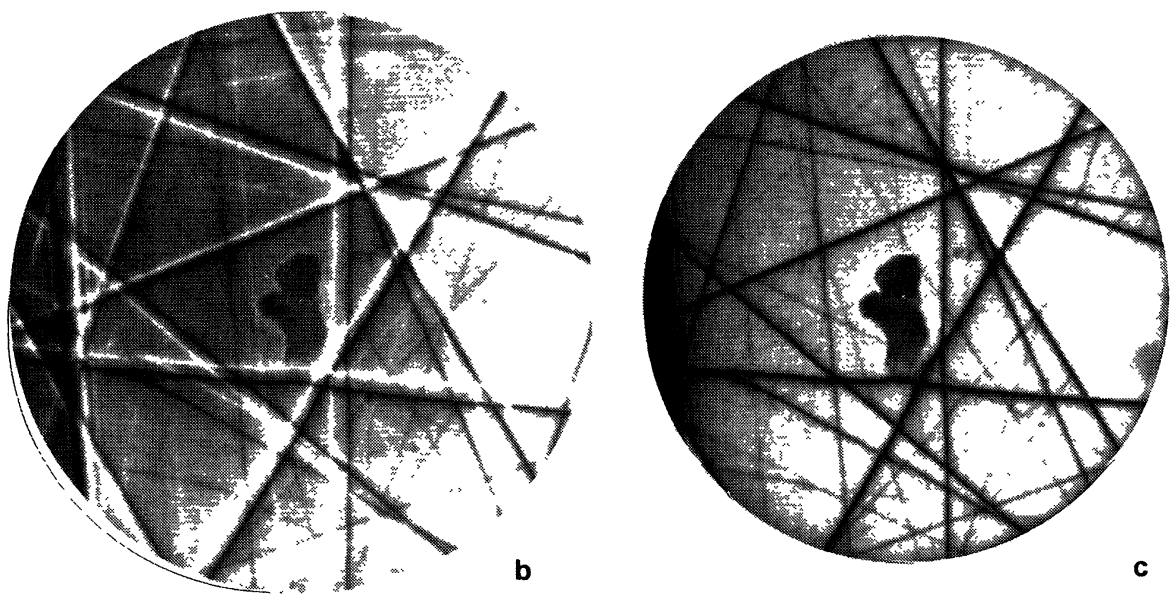

Fig. 2. - Experimental procedure to obtain a CBIM pattern (specimen: silicon) a) The specimen, located at eucentric height is illuminated and focused with an incident parallel beam. b) The incident beam is over or under focused by means of the $\mathrm{C}_{2}$ condenser. Deficiency (dark) and excess (bright) lines appear. Due to spherical aberration, they are not exactly superimposed. c) The objective aperture is used to remove the superimposition of the excess and deficiency lines.

where they form a point diffraction pattern. Since this plane is conjugate to the image plane, a magnified point diffraction pattern is also observed in the image plane. Hence the selected area aperture can be used to isolate and separate the transmitted from the diffracted beams. If the transmitted beams are selected, a "bright field" LACBED pattern is obtained which only displays two high-contrast $h k l$ and $\bar{h} \bar{k} \bar{l}$ deficiency (dark) lines. Alternatively, if the $h k l$ or the $\bar{h} \bar{k} \bar{l}$ diffracted beams are selected, a "dark field" LACBED pattern is observed, only displaying one $h k l$ or $\bar{h} \bar{k} \bar{l}$ excess line.

Because of the large beam convergence, what was just described for one $(h k l)$ lattice plane family occurs simultaneously for many $(h k l)$ families. As a consequence, the bright field LACBED pattern is composed of many deficiency lines, called Bragg lines or Bragg contours.

Figure 1a also shows that the illuminated area ab of the specimen is imaged as $\mathrm{a}^{\prime} \mathrm{b}^{\prime}$ in the back focal plane. This means that the image of the illuminated area is superimposed on the diffraction pattern, with a resolution directly related to the spot size. The resolution of the real space information remains acceptable provided very small spot sizes are used. An image of 
this electron source occurs at $S$, which is conjugate to the electron source. For the over-focus case (not shown) where $S$ occurs above the sample, it is readily seen that the final image will be a shadow image projected from $S$, which limits the image resolution if it is not a point.

\section{Description of the CBIM Technique}

The incident convergent beam is the same as in the case of LACBED, but instead of being focused in the object plane, it is focused above or below it with a defocus value of $\Delta h$ (Fig. $1 \mathrm{~b}$ ). The specimen remains in the object plane of the objective lens, that is to say at the eucentric height.

Transmitted and diffracted beams are generated at $\mathrm{A}^{\prime}$ and $\mathrm{B}^{\prime}$ of the specimen, and as explained above, they produce excess and deficiency lines which are superimposed in the back focal plane.

In the CBIM technique, the screen is now conjugate both to image plane and specimen. This means that an in-focus image $\mathrm{a}^{\prime} \mathrm{b}^{\prime}$ of the illuminated area $\mathrm{ab}$ of the specimen is observed on the image plane and on the screen. In these planes, the excess and deficiency lines are also observed. They exhibit three properties:

- they are not in focus (they are in focus in the back focal plane),

- they have a weight which depends on the spot size, this will be developed later,

- the two $h k l$ excess and deficiency lines are superimposed as well as the two $\bar{h} \bar{k} \bar{l}$ excess and deficiency lines. In LACBED, it is the $h k l$ excess and $\bar{h} \bar{k} \bar{l}$ deficiency lines which are superimposed.

This superimposition can be removed by using the objective aperture situated in the back focal plane of the objective lens.

In conclusion, a LACBED pattern is a line diffraction pattern (similar to a Kossel pattern in X-ray diffraction) with a superimposed image of the illuminated area of the specimen. A CBIM image is an image of the illuminated area of the specimen superimposed with a line diffraction pattern.

\section{Experimental Conditions}

The CBIM patterns were obtained using Philips CM30, Philips CM200 FEG and Zeiss 912 transmission electron microscopes. The nanoprobe mode was used in order to get the large convergences coupled with the small spot sizes required by the CBIM technique. Energy filtered CBIM patterns were obtained with the omega energy filter of the Zeiss 912 microscope and the Gatan Imaging Filter (GIF) fitted on a Philips CM200 microscope. The silicon specimens were prepared by mechanical polishing using the tripod method described by Benedict et al. [14].

A naturally deformed garnet specimen has been used for the identification of the dislocation Burgers vector. Garnet has been chosen because identification of dislocations is difficult in the garnet structure (Rabier et al. [15]). Due to the large unit cell, two-beam diffraction conditions are difficult to obtain, making the conventional method of dislocation characterization based on the $\mathbf{g} \cdot \mathbf{b}=0$ and $\mathbf{g} \cdot \mathbf{b} \times \mathbf{u}=0$ invisibility criteria tedious. Thin sections of the garnet samples $(20 \mu \mathrm{m})$ were prepared by mechanical grinding and then optically polished on both faces with cerium oxide. Thin foils were finally obtained by argon ion milling at $5 \mathrm{kV}$ under a low beam angle of $15^{\circ}$. 


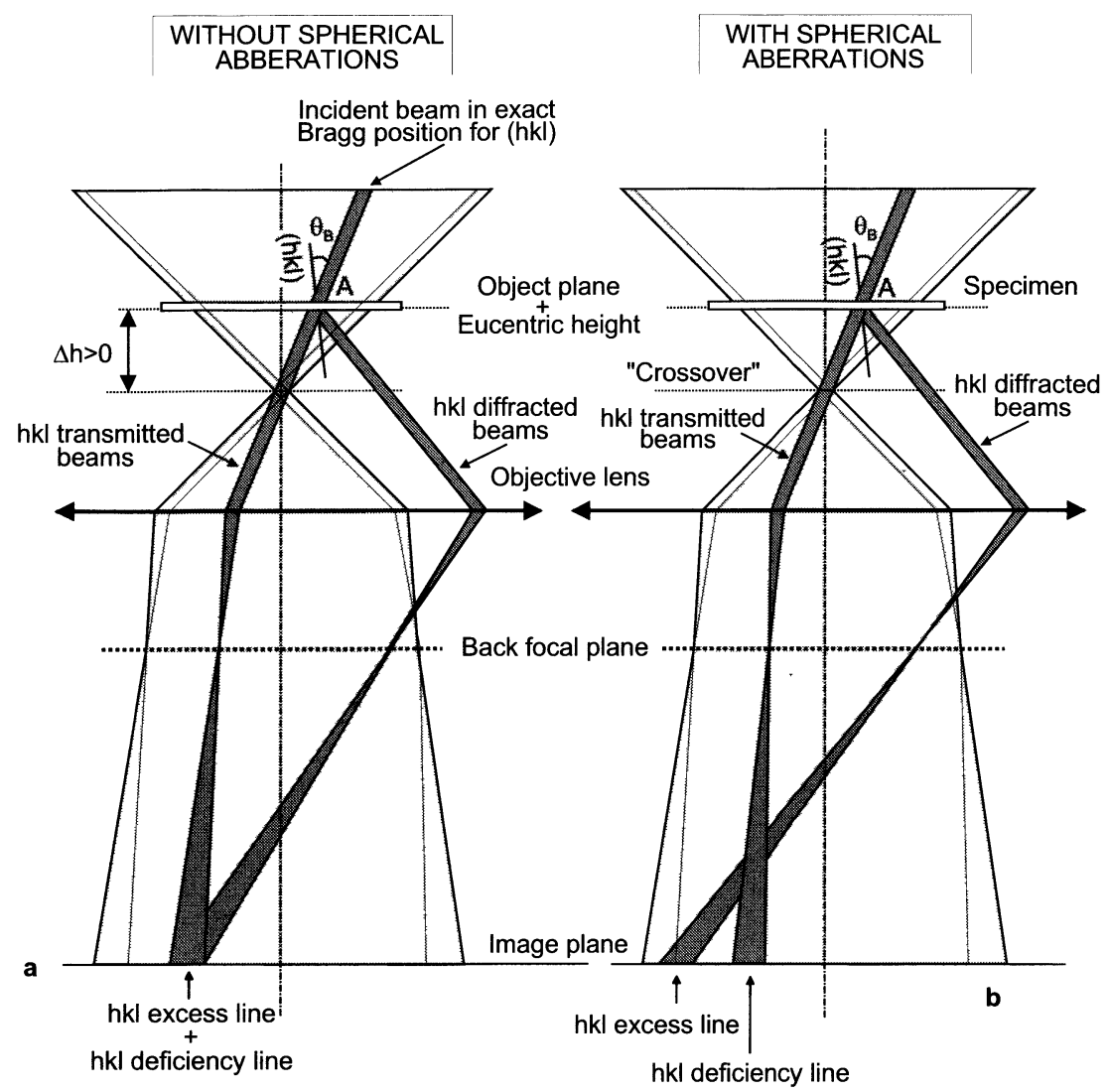

Fig. 3. - The effect of spherical aberration on the superimposition of the $h k l$ excess and deficiency lines. a) Electron paths for a perfect objective lens. The $h k l$ excess and deficiency lines are exactly superimposed in the image plane of the objective lens. b) Electron paths for an objective lens suffering from spherical aberration. Since the $h k l$ diffracted beams are more distant from the optical axis than the transmitted ones, they are more deflected. As a result, the $h k l$ excess and deficiency lines are not exactly superimposed.

The experimental step by step procedure to obtain a CBIM pattern is as follows:

- the specimen is set at the eucentric height of the microscope and the image is focused with a parallel beam in order to set the operating conditions of the objective lens (Fig. 2a).

- the $\mathrm{C}_{2}$ condenser lens is over- or under-focused so as to place the image of the crossover below or above the specimen. Excess and deficiency lines appear as shown in Figure $2 \mathrm{~b}$.

- the objective aperture is used to remove the excess lines (Fig. 2c) so that only the dark deficiency lines remain.

The experimental pattern in Figure 2a exhibits excess and deficiency lines which are not exactly superimposed. This effect is due spherical aberration of the objective lens and can be understood with Figures $3 \mathrm{a}$ and $3 \mathrm{~b}$ displaying the ray paths without and with spherical aberration. Figure $3 \mathrm{~b}$ clearly shows that the transmitted and the diffracted beams originating in point A of the specimen have different angles with respect to the optical axis. Due to spherical aberration they will be focussed differently which explains why they do not superimpose perfectly. 

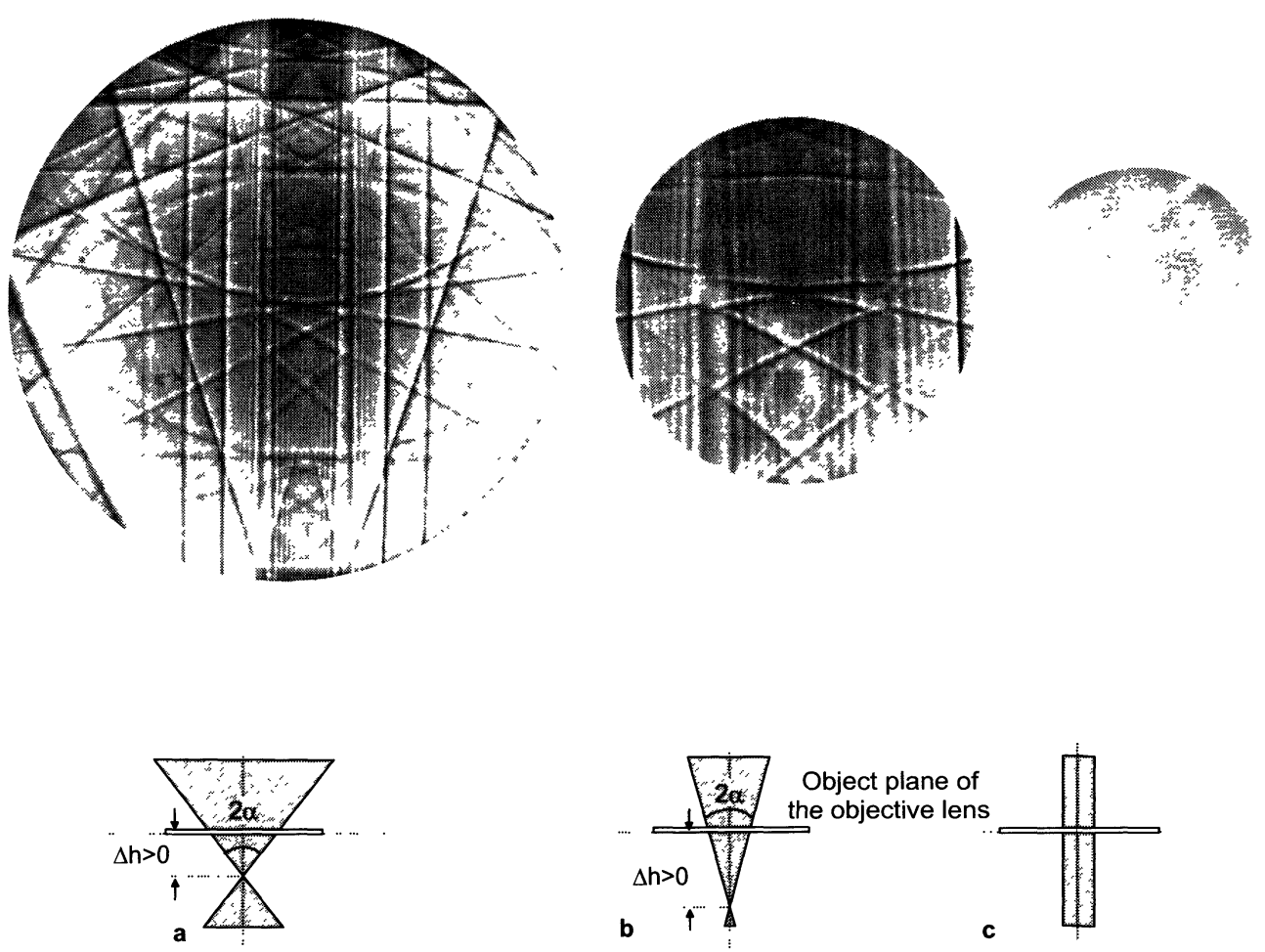

Decreasing of the excitation current of the $\mathrm{C}_{2}$ condenser

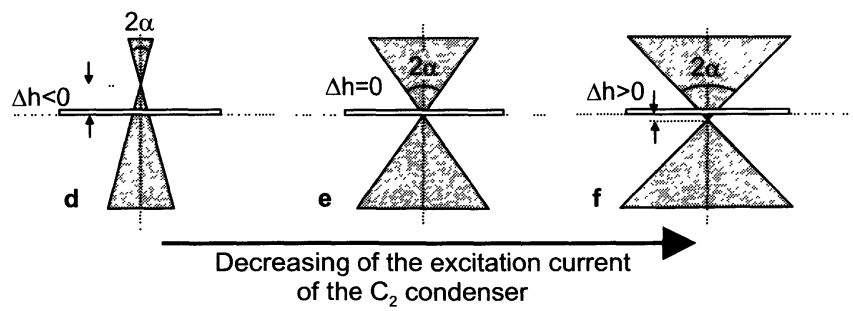

Fig. 4. - Evolution of the CBIM patterns as a function of the excitation current of the $\mathrm{C}_{2}$ condenser. (specimen: silicon near $<001>$ ). a, b) Very strong and strong excitation currents. The incident beam is convergent. The size and the convergence $\alpha=1.7^{\circ}$ for a and $0.6^{\circ}$ for $\mathrm{b}$ ) of the CBIM patterns are large. c) Medium excitation current. The incident beam is parallel and hence excess and deficiency lines are not present. d) Low excitation current. The incident beam is divergent. The size and the convergence $\left(\alpha=0.5^{\circ}\right)$ of the CBIM pattern is small. e, f) Weak and very weak excitation currents. The convergent beam is focused or under focused. The size of the CBIM pattern is to small to be useful. 


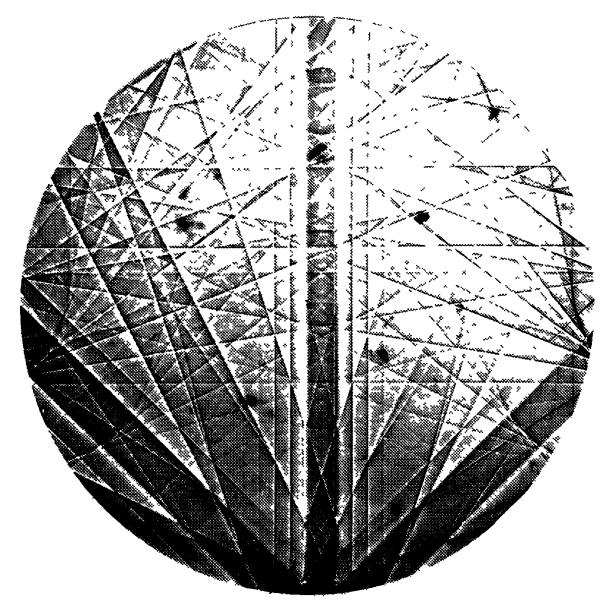

Fig. 5. - CBIM pattern obtained with a very strong excitation current of the $\mathrm{C}_{2}$ condenser lens on a Philips CM30 electron microscope. The convergence semi-angle $\alpha$ is about $6^{\circ}$ (specimen: silicon near $<001>$ ).

\section{Effects of the Different Experimental Parameters}

\subsection{Effect of the $\mathbf{C}_{2}$ condenser Lens Focus}

Figure 4 gives the whole evolution of the CBIM patterns as a function of the excitation current of the $\mathrm{C}_{2}$ condenser lens.

When the lens current is decreased, the convergence angle $\alpha$, the defocus value $\Delta h$ and the size $S$ of the illuminated area, that is the size of the CBIM pattern, are modified. Useful CBIM images are only obtained with strong excitations, around the parallel illumination condition (Figs. 4a to d). Low excitation currents give CBIM patterns with sizes too small to be useful (Figs. 4e and f). Of course, no lines are observed for a parallel beam.

The convergence semi-angle $\alpha$ can be very large for high excitation currents. The maximum value obtained with the "twin" lens of a Philips CM30 microscope is about $6^{\circ}$ (Fig. 5 ), in which case the acronym LACBIM (Large Angle CBIM) would be best suited.

The magnification of the CBIM pattern depends on the defocus value $\Delta h$. However this parameter has no influence on the magnification of the superimposed image. This property is opposite to the one observed for LACBED patterns where $\Delta h$ had an effect on the magnification of the image and no effect on the diffraction pattern.

Changing the sign of the defocus $\Delta h$ produces a $180^{\circ}$ rotation of the diffraction pattern (Fig. 6). With the LACBED patterns, it was the image which was rotated and not the diffraction pattern.

\subsection{Effect of the Spot Size}

The spot size $S$ has a direct effect on the width of the excess and deficiency lines as shown in Figure 7 .

For that reason, it is essential to use very small spot sizes. Spot sizes above $50 \mathrm{~nm}$ give very poor patterns. The spot size has no effect on the image of the illuminated area. 

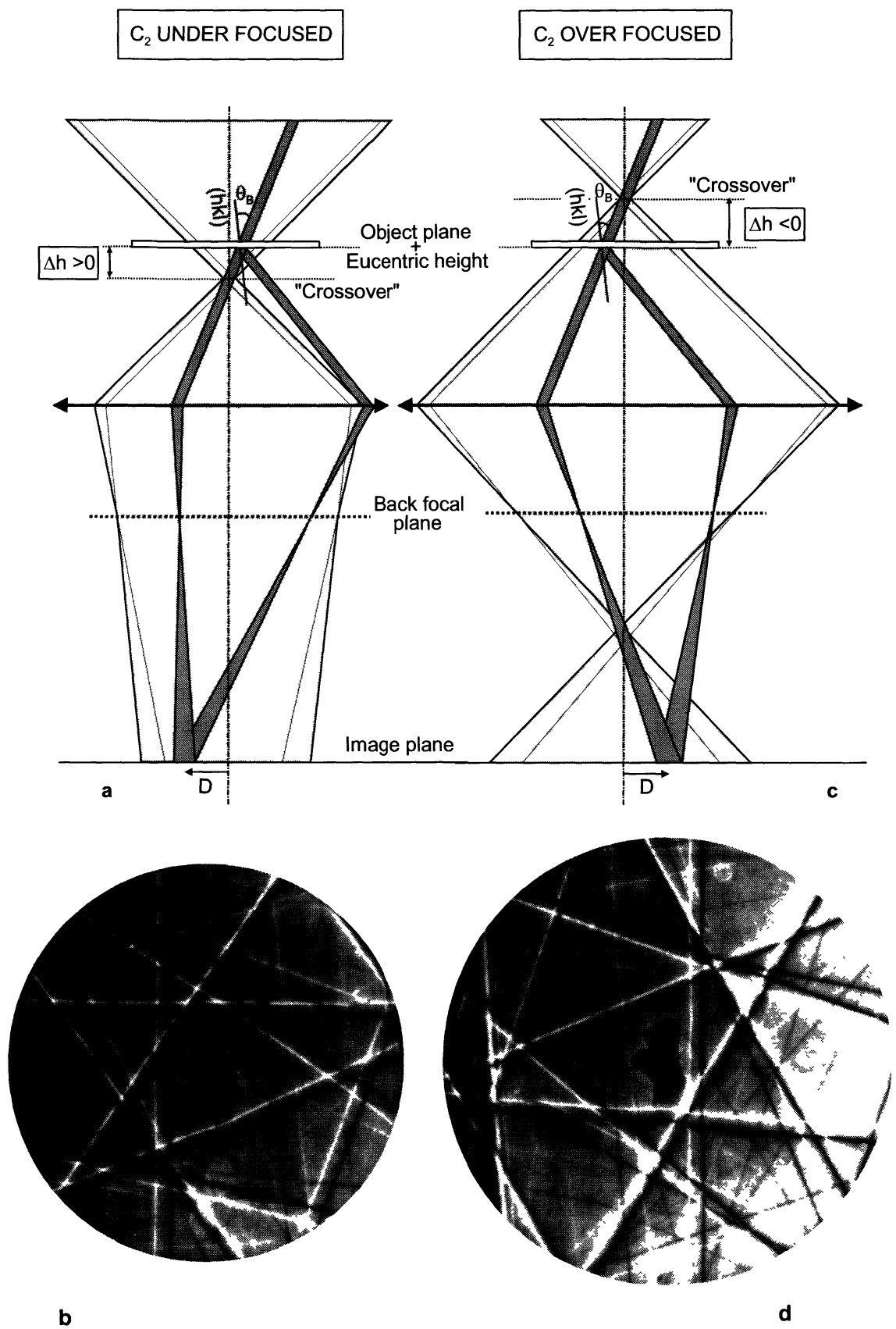

b

Fig. 6. - Effect of the sign of the $\Delta h$ defocus on the CBIM patterns. (specimen: silicon). a, b) The $\mathrm{C}_{2}$ condenser is under focus $\left.(\Delta h>0) . \mathrm{c}, \mathrm{d}\right)$ The $\mathrm{C}_{2}$ condenser is over focus $(\Delta h<0)$. A $180^{\circ}$ rotation of the CBIM pattern is observed. 

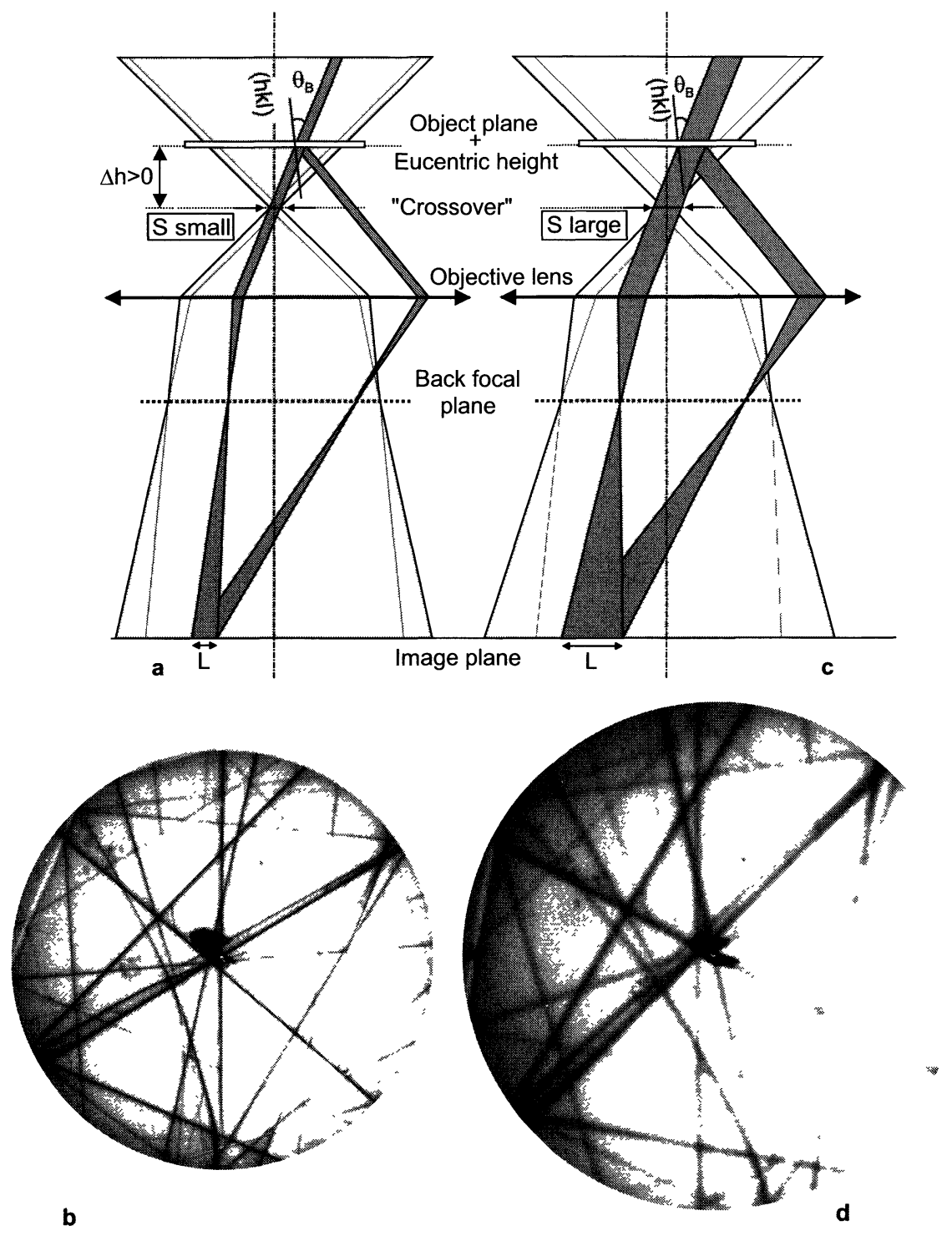

Fig. 7. - Effect of the spot size $S$ on the CBIM patterns. (specimen: silicon). a, b) Small spot size (1.2 $\mathrm{nm}$ for the experimental pattern). The excess and deficiency lines are sharp. c, d) Large spot size (25 $\mathrm{nm}$ for the experimental pattern). The excess and deficiency lines are broad.

\subsection{Effect of the Objective Aperture}

The objective aperture has three main effects (Fig. 8):

- it removes the superimposition of the excess and deficiency lines;

- it produces an angular filtering of the inelastic electrons;

- it reduces the beam convergence. 

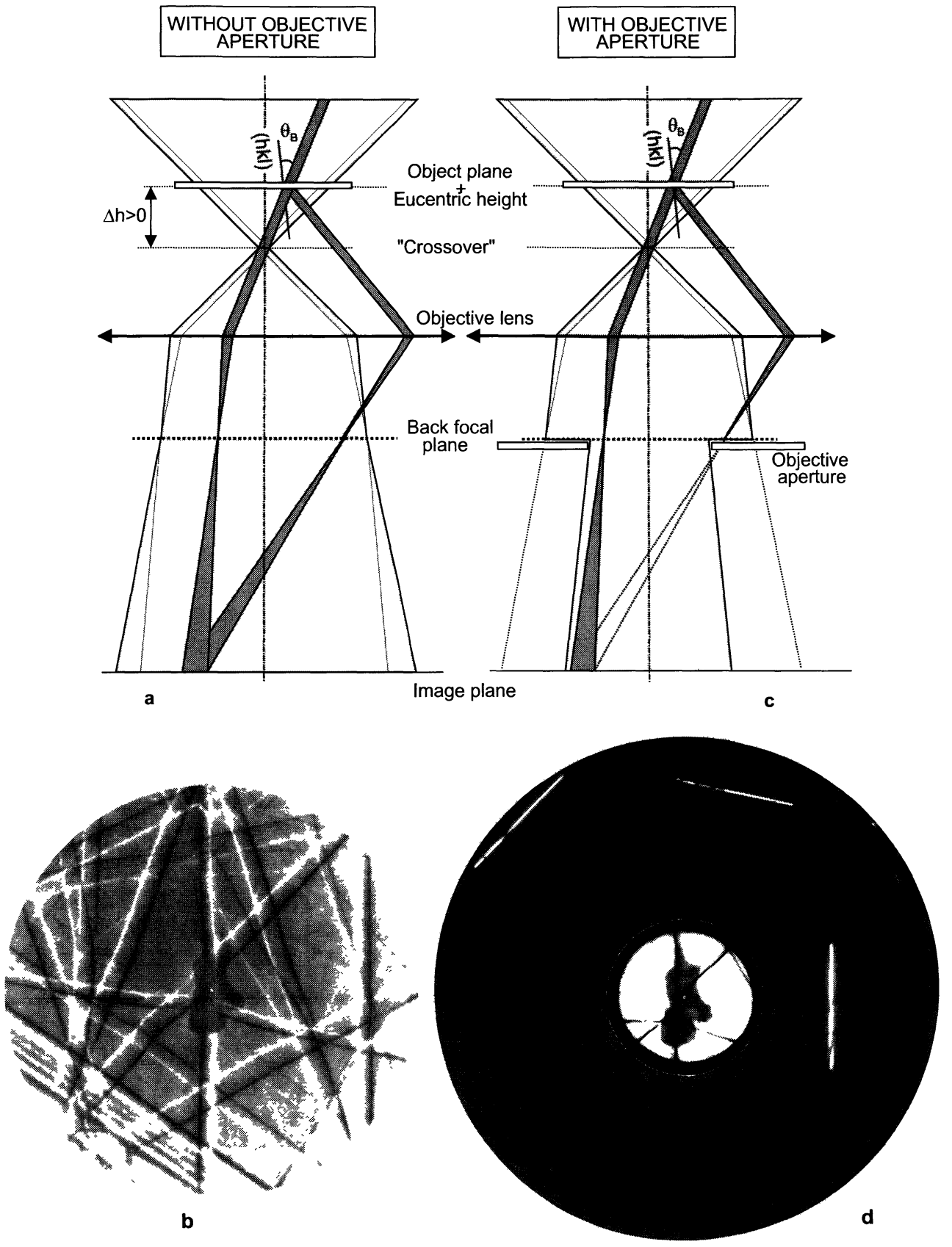

Fig. 8. - Effect of the objective aperture. Silicon specimen. a, b) Electron paths and CBIM pattern without objective aperture. The excess and deficiency lines are superimposed. c, d) CBIM electron paths when an objective aperture is used. The superimposition is removed and the convergence is decreased. The quality of the pattern is improved due to angular filtering of the objective aperture. 

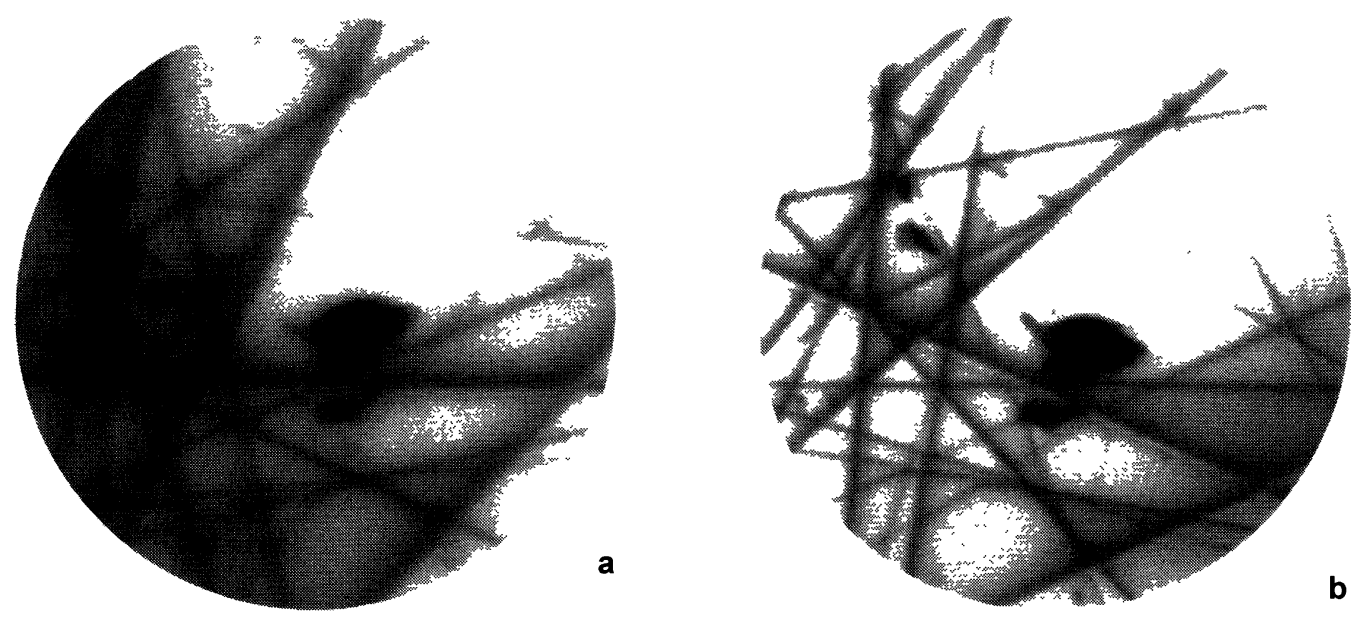

Fig. 9. - Effect of energy filtering on CBIM patterns (specimen: silicon). a) Non filtered pattern. b) CBIM pattern formed with the "omega" filter of the Zeiss 912 electron microscope. A sensible improvement of the pattern quality is visible.

\section{Advantages and Disadvantages of the CBIM Techique}

CBIM is very easy to perform, starting from a conventional parallel beam observation, $\mathrm{C}_{2}$ is slightly decreased or increased. Since it is a defocus method, it is very well suited for the analysis of defects, an aspect which will be developed in the second part of this paper.

The quality of CBIM patterns is not as good as the quality of LACBED patterns, because the angular filtering of the objective aperture is not as efficient as the angular filtering of the selected area aperture in the case of LACBED patterns [7]. In order to keep a reasonable convergence in a CBIM pattern, the size of the objective aperture should remain large. This poor angular filtering of the objective aperture can be greatly enhanced by using an energy filter. Examples of energy filtered CBIM patterns are given in Figures $9 \mathrm{~b}$ and $13 \mathrm{a}$, b demonstrating that filtered CBIM patterns are nearly as good as LACBED patterns.

\section{Application of the CBIM Technique to the Characterization of the Burgers Vectors of Dislocations}

LACBED and CBIM share the property of combining real and reciprocal space information and in the case of CBIM, the specimen is located at the eucentric position. This suggests that CBIM can be used, similarly as LACBED, to characterize dislocation Burgers vectors.

Figure 10a shows a CBIM image obtained from a garnet specimen without an objective aperture and with a convergence of about $3.5^{\circ}$. The edge of the thin foil and a crack running through the specimen are clearly visible as well as deficiency and excess lines. These deficiency and excess lines do not superimpose exactly for the reason explained above. The lines can be indexed using the simulated pattern of Figure 10b. The beam stop is pointed at a dislocation and one can see the effects of the strain field on the -3-41 line (see enlargement in Figure 10c taken with an objective aperture). Similarly as in LACBED, the line is split and twisted at the intersection with the dislocation line, which indicates that $\mathbf{g} \cdot \mathbf{b}=3$. However, a complete Burgers vector determination requires the dislocation line to cross at least three independent 


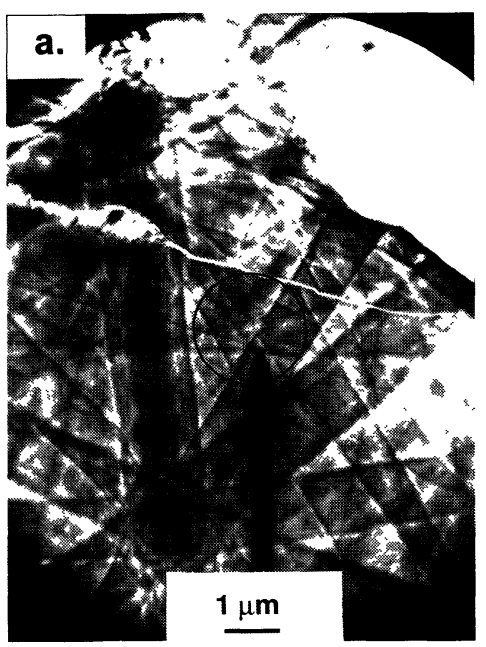

b.
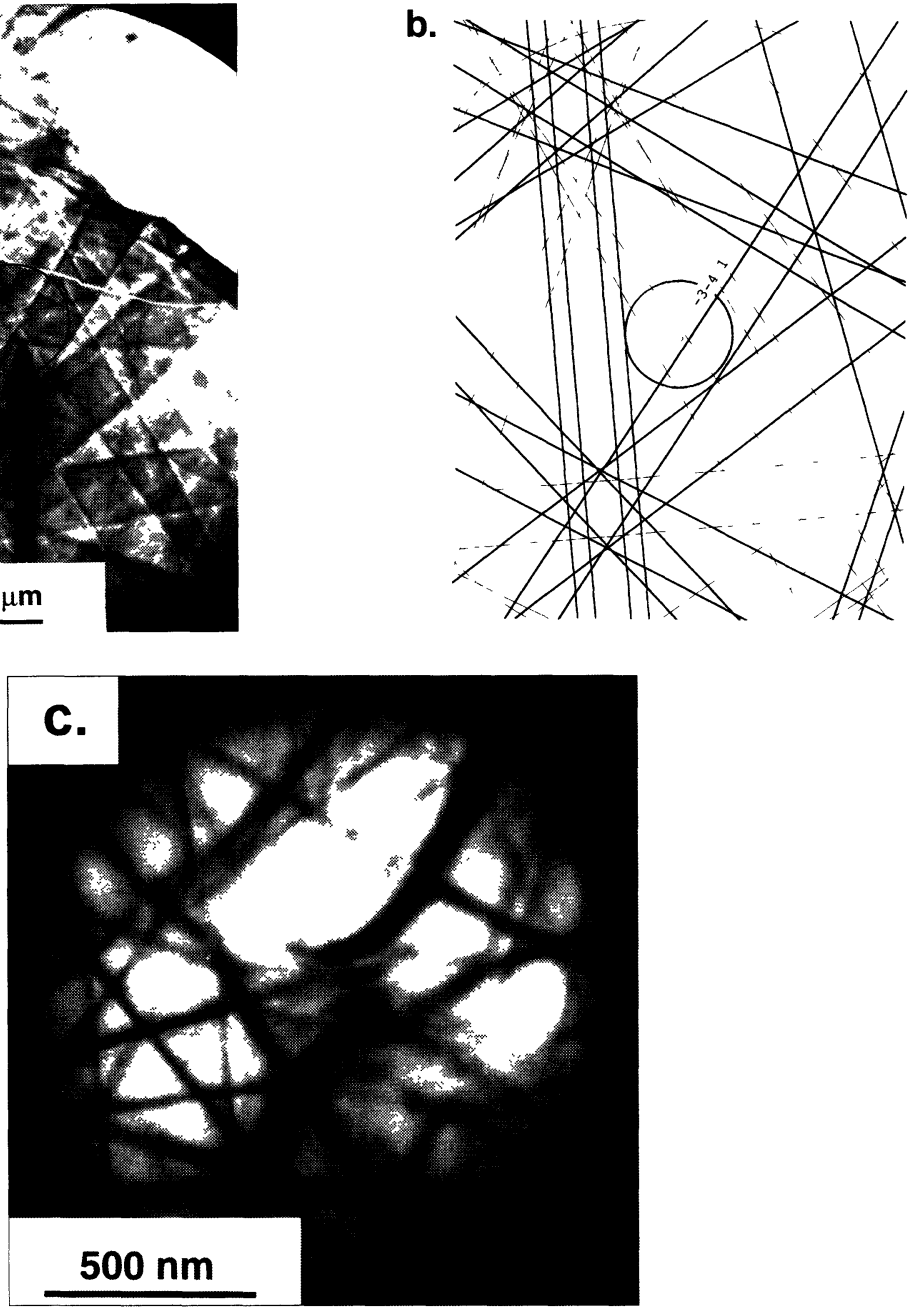

Fig. 10. - a) CBIM image of a garnet specimen. Note the edge of the foil and the crack running through the specimen. Convergence semi-angle: $3.5^{\circ}$. b) Corresponding simulated CBIM pattern. Simulation obtained with "Electron Diffraction" (J.P. Morniroli, D. Vankieken and L. Winter). c) Enlargement of Figure 10 a showing the effect of the dislocation line on the $\overline{3} \overline{4} 1$ deficiency line. The excess lines have been suppressed by adding a small objective aperture. The convergence semi-angle is reduced to about $0.3^{\circ}$.

deficiency lines. The Burgers vector indices are then obtained by solving the $\mathbf{g}_{i} \cdot \mathbf{b}=n_{i}$ linear system. Depending on the dislocation line's length, two methods can be used. If the dislocation line is long enough, it can be placed so that it crosses simultaneously three different deficiency lines, otherwise the dislocation line must be placed successively on three different deficiency lines. Figure 11 shows such an experiment performed on garnet. The dislocation Burgers vector can be unambiguously identified as $1 / 2$ [111]. 

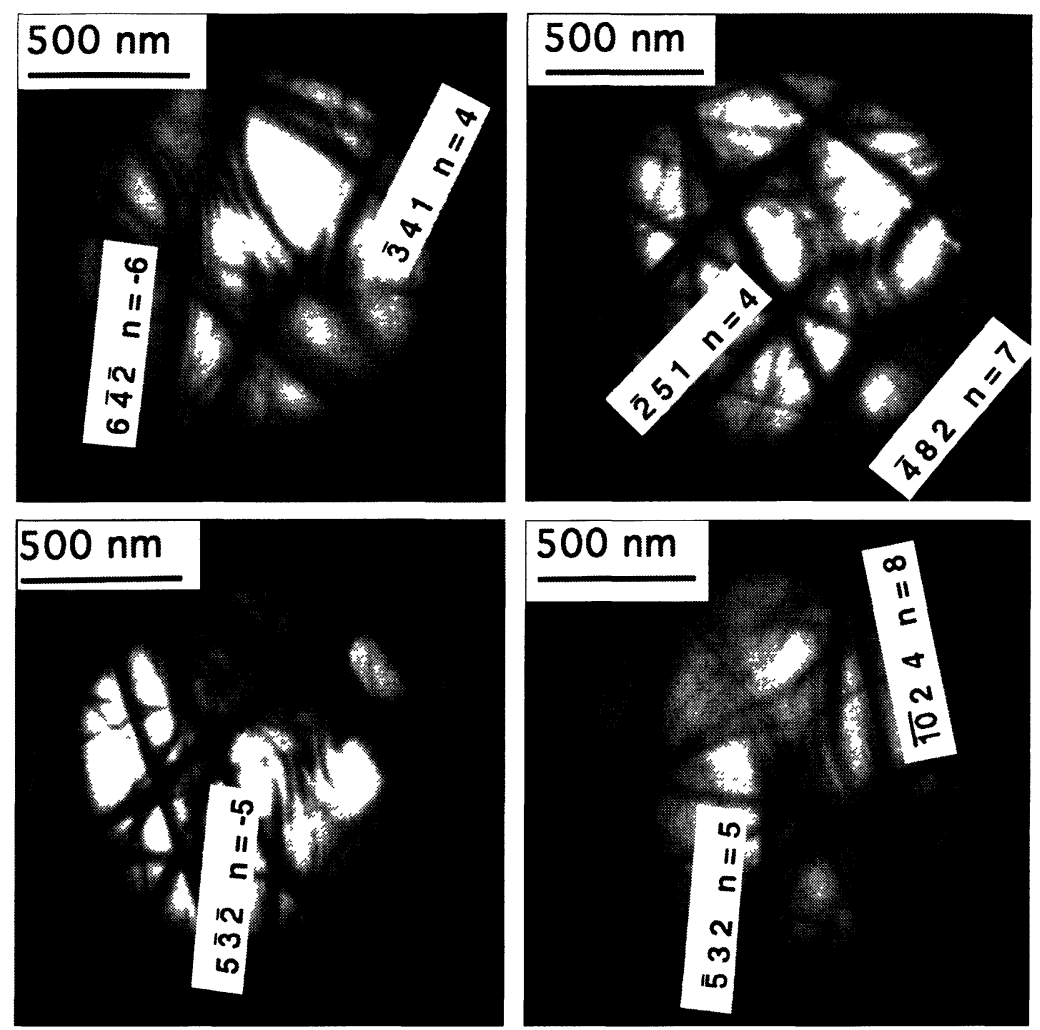

Fig. 11. - Characterization of a dislocation in garnet. The dislocation line has been successively crossed with seven deficiency lines. The Burgers vector is $1 / 2$ [111].
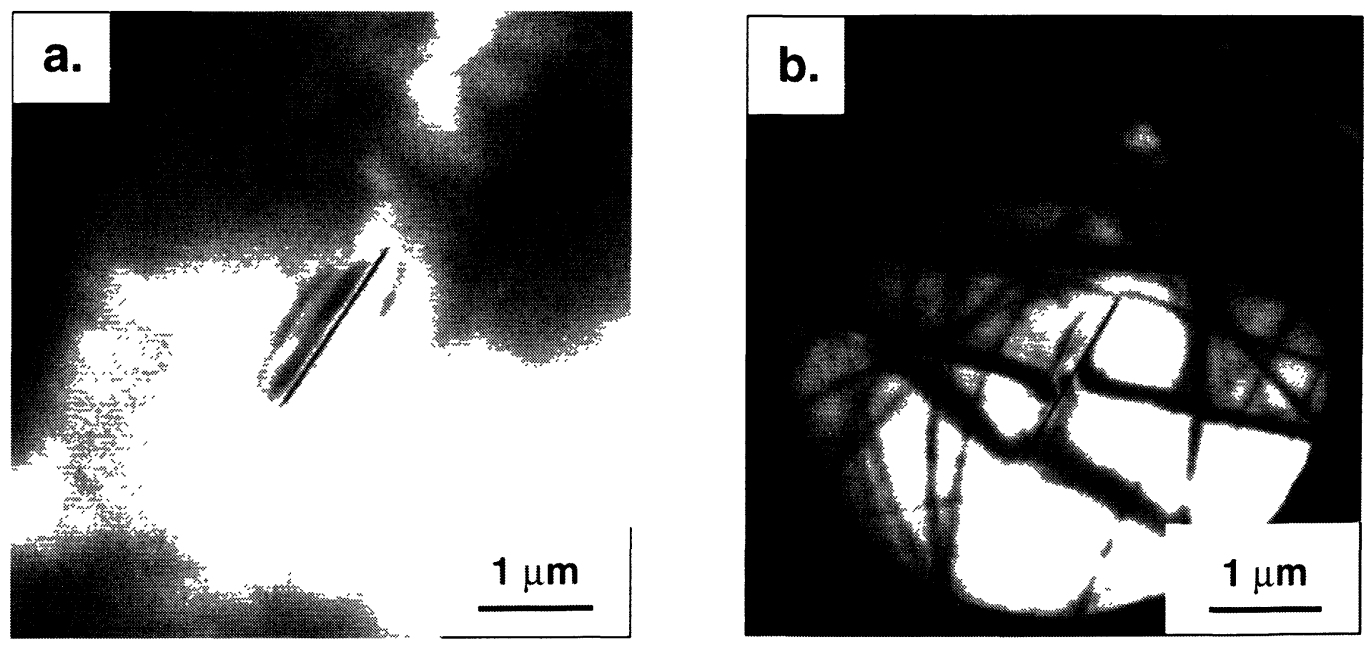

Fig. 12. - a) Bright field image of a dislocation in garnet. Parallel beam in nanoprobe mode. b) Same dislocation. CBIM image obtained by adjusting the $\mathrm{C}_{2}$ current so as to get a convergent beam. 

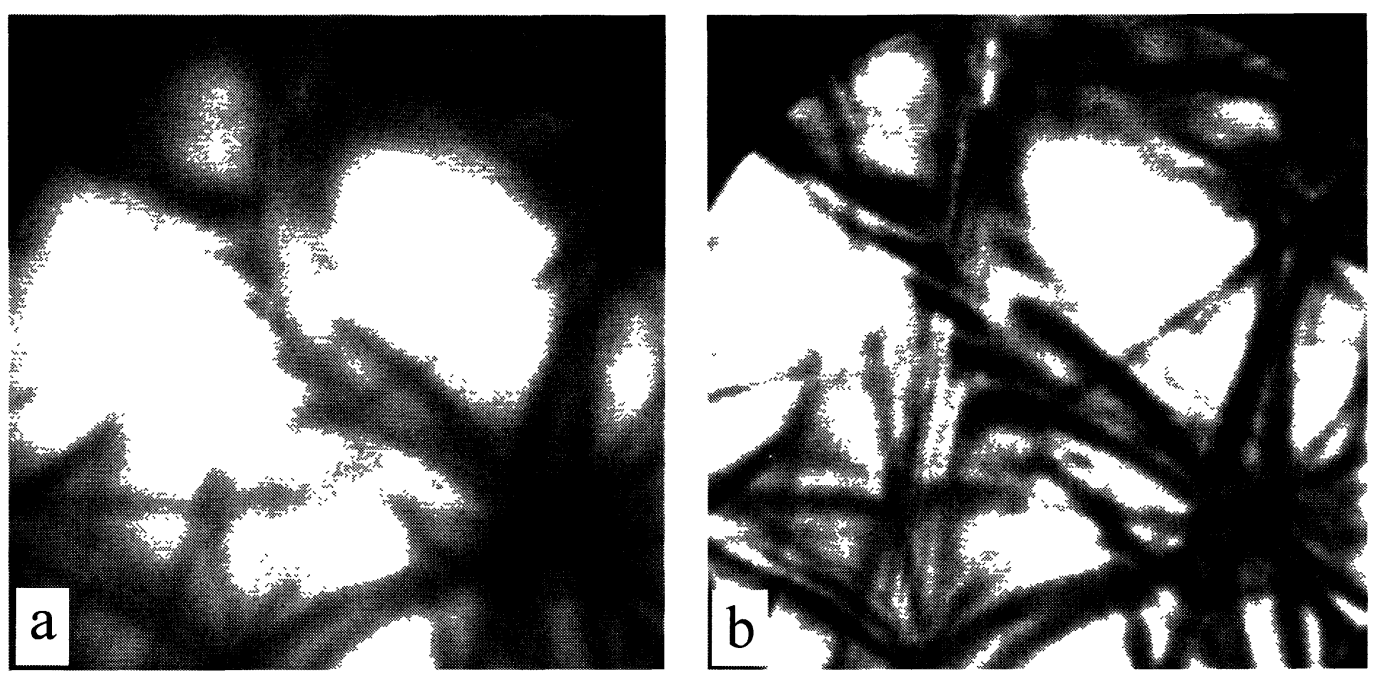

Fig. 13. - a) CBIM image of the effect of a dislocation in a thick zone of the foil. Unfiltered image. b) Corresponding filtered image using a GIF with a $10 \mathrm{eV}$ slit.

\section{Discussion and Conclusion}

Dislocation characterization is one of the most successful applications of the LACBED technique. The previous section demonstrates that the CBIM technique is also potentially attractive for characterizing crystal defects and we shall now discuss some practical aspects related to the use of CBIM and LACBED.

The most obvious advantage of using CBIM is the fact that it is an image mode, so the specimen is in focus and placed at the eucentric height. This allows a much better (and easier) control of the microstructure while operating the TEM which may turn out to be crucial if the dislocation microstructure is complicated (e.g. several dislocation types) and when a given defect has to be analysed. The contrast of dislocations, in CBIM as well as in conventional TEM, depends on the diffracting conditions i.e. on the line which crosses with the dislocation. The dislocation lines in Figures 10 and 11 are invisible, whereas in the example of Figure 12 the dislocation line is clearly visible. The salient point is that it is very easy and fast to switch between CBIM and a conventional bright field image. This is shown in Figure 12 where the only difference between $12 \mathrm{a}$ and $12 \mathrm{~b}$ is the $\mathrm{C}_{2}$ excitation current. It is therefore very easy to check at any time whether the desired defect is still analysed.

The disadvantage of CBIM is that the diffraction pattern associated with the image is not of the same high quality as in LACBED patterns. The deficiency and or excess lines usually appear much broader in CBIM than in LACBED. The width of the CBIM lines depends on the spot size, so it is possible to get thinner lines by decreasing the spot size, but this is limited by brightness since the image becomes darker. Another reason for the high quality of LACBED patterns is the efficient filtering of inelastically scattered electrons by the selected area aperture. In CBIM the effect of the objective aperture is less efficient but a similar result can be obtained with an energy filter. An example of zero-loss filtering acquired on a GIF, is shown in Figure 13 where the visibility of the fringes is dramatically improved by filtering with a $10 \mathrm{eV}$ slit. 
We have outlined the fact that CBIM is a large angle convergent beam method (Fig. 10a), however the use of an objective aperture significantly reduces the width of the diffraction pattern (Fig. 10b). Without the objective aperture, superposition of excess and deficiency lines results in a very faint contrast on the screen of the microscope (it is more visible on the micrographs and can be reinforced on the prints). Low-contrast patterns may be difficult to interpret at the microscope. If we introduce an objective aperture, the visibility of the lines is much better, however the smallness of the visible field renders identification of the lines more difficult.

For both methods, the quality of the patterns can be improved by using a smaller source, such as that obtainable on dedicated cold field-emission STEM instruments. As an example, we cite the work of $\mathrm{Zhu}$ and Cowley [16], on antiphase domains in $\mathrm{Cu}_{3} \mathrm{Au}$, using sub-nanometer probe.

In conclusion the CBIM technique appears to be very promising for crystal defect characterization studies. Notwithstanding the fact that here we only presented an application on dislocations, we strongly believe that CBIM can also be applied to a much broader class of defects (partial dislocations, grain boundaries, twins, stacking faults, ....). LACBED and CBIM are complementary techniques, and depending on the exact application, one of them should be favoured.

\section{Acknowledgments}

Work at Tempe is supported by N.S.F. award DMR9412146.

\section{References}

[1] Tanaka M., J. Electron Microsc. 29 (1980) 408-412.

[2] Cherns D. and Preston A.R., Proc. XIth Int. Cong. on Electron Microscopy, Kyoto (1986) pp. 721-722.

[3] Carpenter R., Spence J.C.H., Acta Cryst. A38 (1982) 55-68.

[4] Tanaka M., Terauchi M., Kaneyama T., Convergent Beam Electron Diffraction, Vol. 2 (Jeol Ltd, Tokyo, 1988).

[5] Cherns D., Morniroli J.P., Ultramicroscopy 53 (1994) 167-180.

[6] Morniroli J.P. and Cherns D., Ultramicroscopy 62 (1996) 53-63.

[7] Ya Y.f., Wan R.H., Fen J.L., Phil. Mag. Lett. 66 (1992) 197-201.

[8] Jesson D.E., Steeds J.W., Ultramicroscopy 31 (1989) 399-430.

[9] Spence J.C.H., Zuo J.M., Electron Microdiffraction (Plenum, 1992).

[10] Jordan I.K., Rossouw C.J., Vincent R., Ultramicroscopy 35 (1991) 237- 243.

[11] Xin Y., Duan X.F., Ultramicroscopy 53 (1994) 159-165.

[12] Humphreys C.J., Maher D.M., Frazer H.L., Eaglesham D., Phil. Mag. 58 (1988) 787-798.

[13] Humphreys C.J., Eaglesham D., Maher D.M., Frazer H.L., Ultramicroscopy 26 (1988) 13-24.

[14] Benedict J.P., et al., in: Specimen Preparation for transmission electron microscopy of materials - II, R. Anderson, Ed., MRS Symp. Proc. 199 (1990) 189.

[15] Rabier J., Garem H., Veyssière P., J. Appl. Phys. 47 (1976) 4755-4758.

[16] Zhu J., Cowley J.M., Acta Cryst. A38 (1982) 718-728. 